\title{
Macrophages Are Required for Influenza Virus Infection of Human Lymphocytes
}

\author{
David J. Mock, Frank Domurat, Norbert J. Roberts, Jr., Edward E. Walsh, Mark R. Licht, and Peter Keng \\ Infectious Diseases Unit, Department of Medicine, and the Cancer Center, University of Rochester School of Medicine, \\ Rochester, New York 14642
}

\begin{abstract}
Monocyte and lymphocyte surface-expressed viral antigens have been demonstrated after exposure of unseparated human mononuclear leukocytes to influenza virus in vitro. The current studies, using [ ${ }^{35}$ S]methionine pulse-labeled purified preparations of virusexposed macrophages, depleted of lymphocytes, demonstrate that the presence of these viral proteins does represent new synthesis. However, purified lymphocytes, depleted of monocytes-macrophages and exposed to influenza virus, showed no detectable viral protein synthesis. In further experiments, unseparated mononuclear leukocytes were exposed to virus and subsequently separated by countercurrent centrifugal elutriation. Both macrophages and lymphocytes were then shown to synthesize influenza proteins. Cell-free control or influenza virus-infected macrophage-derived supernatant fluids did not facilitate influenza virus infection of the lymphocytes. The data suggest that macrophages are required for influenza virus infection of human lymphocytes, and raise the possibility that macrophage facilitation of an abortive infection of lymphocytes plays a role in the generation of effective immunity to viral antigens.
\end{abstract}

\section{Introduction}

In our previous studies, we showed that exposure of human mononuclear leukocytes to influenza virus in vitro resulted in depressed lymphocyte proliferative responses to mitogens (1). The depressed proliferative responses were further shown to be due to a primary alteration in monocyte-macrophage accessory cell function after exposure to virus; lymphocyte proliferation in response to mitogens remained intact in the presence of unexposed monocytes-macrophages $(1,2)$. Additional studies demonstrated the production of significant quantities of immunoregulatory factors, notably both interferon and interleukin 1 (IL-1), ${ }^{1}$ by the influenza virus-exposed monocytes-macrophages $(3,4)$. No interferon was produced by virus-exposed purified lymphocytes.

Address reprint requests to Dr. Roberts, Infectious Diseases Unit, University of Rochester School of Medicine, Box 689, 601 Elmwood Avenue, Rochester, NY 14642.

Received for publication 8 May 1986.

1. Abbreviations used in this paper: HA, hemagglutinin; IL-1, interleukin 1; M, matrix protein; NA/NP, neuraminidase/nucleoprotein; NSE, nonspecific esterase.

J. Clin. Invest.

(C) The American Society for Clinical Investigation, Inc.

0021-9738/87/02/0620/05 \$1.00

Volume 79, February 1987, 620-624
The results of these studies provided evidence that influenza virus interacts with human macrophages but provided no evidence of a primary interaction of the virus with lymphocytes. Therefore, the possibility remained that human lymphocytes are not infected by the viruses. However, in subsequent experiments, using fluorescein-conjugated anti-influenza antibodies, both monocytes-macrophages and lymphocytes were found to express influenza virus antigens on their surfaces after exposure of unseparated mononuclear leukocytes to infectious virus (5). Nonetheless, the possibility that input viral antigens were merely processed and expressed by the leukocytes could not be excluded. Therefore, the current studies were undertaken to determine whether or not human monocytes-macrophages and lymphocytes, exposed to influenza virus, synthesize new influenza proteins as an indication of active cell infection. In reporting these studies, we describe a novel accessory cell function of monocytesmacrophages that is related to the synthesis of influenza virus antigens by autologous lymphocytes.

\section{Methods}

Cell collection and separation. Mononuclear leukocytes were obtained from the peripheral blood of healthy volunteers by Ficoll-Hypaque sedimentation. Purified monocyte-derived macrophages were obtained by adherence to plastic Petri dishes for $24 \mathrm{~h}$ to $7 \mathrm{~d}$, with extensive washing to remove nonadherent cells $(1,2)$. The resultant adherent cell population contained $>97 \%$ macrophages by nonspecific esterase (NSE) staining $(1,3)$. Nonadherent cells, containing $97-99.5 \%$ lymphocytes by morphologic criteria and NSE stains, were obtained by each of three separate techniques: (a) multiple passes of cells in plastic dishes, with the nonadherent cells gently removed to new dishes every $24 \mathrm{~h}$ for a total of four times; although the least efficient of the three, this procedure consistently yielded > 97\% pure lymphocytes; $(b)$ 24-h adherence of leukocytes using plastic dishes, followed by passage of nonadherent cells through nylon wool columns $(1,3)$; the eluate contained $>99.5 \%$ pure lymphocytes; and $(c)$ countercurrent centrifugal elutriation $(6,7)$; fractions were collected that contained $>99.5 \%$ pure lymphocytes.

Exposure to virus and radiolabel. Cells (unseparated mononuclear leukocytes, macrophages, and lymphocytes) were exposed or sham-exposed to influenza A/AA/Marton/43 H1N1, A/Japan/305/57 H2N2, or A/Scotland/840/74 $\mathrm{H} 3 \mathrm{~N} 2$ for $1 \mathrm{~h}$ at $37^{\circ} \mathrm{C}(3-5)$ in serum-free medium 199. Cells were exposed at multiplicities of infection ranging from 0.1 to 10. Lymphocytes were exposed to virus in the absence and in the presence of trypsin $(8,9)$. Cells were then washed and incubated for various further time intervals $(0-72 \mathrm{~h})$, at the end of which the medium was removed and replaced for $2 \mathrm{~h}$ with methionine-free medium supplemented with $50 \mu \mathrm{Ci}$ of $\left.{ }^{35} \mathrm{~S}\right]$ methionine. Cells were then washed and lysed with an SDS-containing detergent buffer for subsequent analysis by SDS polyacrylamide gel electrophoresis (PAGE) and autoradiography (10). Immunoprecipitation of synthesized proteins was performed using $\mathrm{NIH}$ reference antisera directed against influenza hemagglutinin (anti$\mathrm{H} 1$; V-314-511-517), neuraminidase (anti-N1; V-308-513-157), and matrix protein (anti-M; V-306-501-157). 


\section{Results}

Purified monocyte-derived macrophages obtained by adherence separation were exposed to influenza virus. The cells were pulsed with $\left[{ }^{35} \mathrm{~S}\right]$ methionine after varying intervals, for kinetic analysis of synthesis of influenza virus-directed and host-directed protein synthesis. The interval between phlebotomy, for collection of peripheral blood-derived monocytes-macrophages, and in vitro exposure or sham-exposure of the cells to virus varied from $4 \mathrm{~h}$ to $7 \mathrm{~d}$. Results using 18-h cultured cells (Fig. 1) are representative of data derived from exposure of monocytes-macrophages to virus at all other intervals after collection.

Three proteins, with molecular masses of $\sim 85,60$, and 25 $\mathrm{kD}$, were present in lysates of influenza virus-infected macrophages but absent from corresponding control cells (Fig. $1 a$ ). The proteins represented influenza hemagglutinin (HA), neuraminidase/nucleoprotein (NA/NP), and matrix protein (M), respectively, as determined by immunoprecipitation with specific antisera (Fig. $1 \mathrm{~b}$ ). Viral antigen synthesis was present as early as $2 \mathrm{~h}$ after infection, appeared to be maximal from 2 to $6 \mathrm{~h}$ after infection, and diminished thereafter. The results shown are also representative of data from exposure of the cells to other subtypes/strains of influenza virus (A/Japan/305/57 H2N2 and A/Scotland/840/74 H3N2). Viral antigen synthesis was seen with multiplicities of infection ranging from 0.1 to 10 . Exposure of cells to heat-inactivated influenza $A$ virus yielded results identical to those observed in control lanes (data not shown).

Purified lymphocytes obtained by each of the several separation techniques were exposed or sham-exposed to influenza virus, washed, and incubated for various further intervals ranging

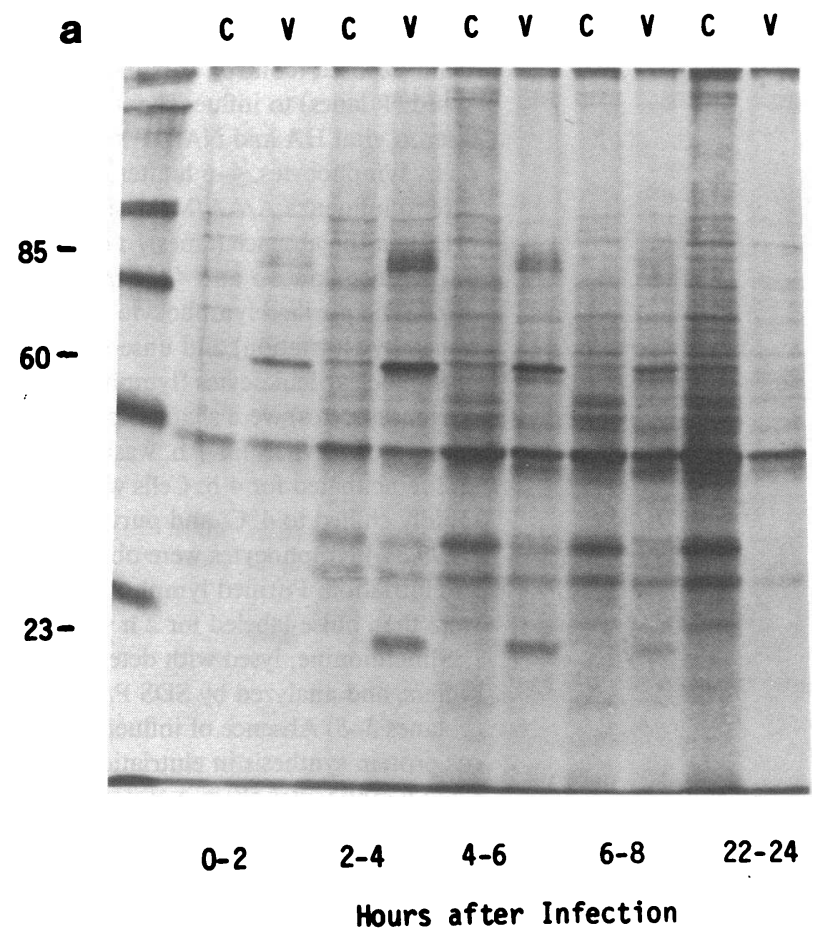

Figure 1. (a) Autoradiograms of purified human peripheral blood-derived monocytes-macrophages (sham-exposed, $C$ lanes) or exposed ( $V$ lanes) to influenza A/AA/Marton/43 $\mathrm{H} 1 \mathrm{~N} 1$ at a multiplicity of infection of 1.0. Each lane represents lysates from $2 \times 10^{6}$ cells. Cells were pulsed, for the indicated intervals after exposure to virus, with

${ }^{35}$ S $]$ methionine. (b) Immunoprecipitation of lysates from either sham- from 0 to $72 \mathrm{~h}$. At the end of each interval, lymphocytes were pulsed with $\left[{ }^{35} \mathrm{~S}\right] \mathrm{methionine,} \mathrm{washed,} \mathrm{and} \mathrm{lysed} \mathrm{for} \mathrm{analysis} \mathrm{by}$ SDS PAGE autoradiography. Fig. 2 shows samples pulsed from 4 to $6 \mathrm{~h}$. No influenza virus proteins could be detected in lysates from purified lymphocyte populations after exposure to $\mathrm{H} 1 \mathrm{~N} 1$ (shown in Fig. 2), or H2N2, or H3N2 strains of influenza virus, with lysates from lymphocytes exposed at multiplicities of infection varying from 0.1 to 10 (data are shown in Fig. 2 for MOI $=1.0$ ). The addition of trypsin to the medium during exposure of lymphocytes to virus $(8,9)$ had no effect on lymphocyte infectivity.

The data suggested that, while purified preparations of human macrophages exposed to influenza $A$ virus in vitro produced new viral antigens, purified lymphocytes similarly exposed did not. Previous studies, however, had demonstrated surface viral antigen expression, using immunofluorescence assays, by both lymphocytes and macrophages (exposed as unseparated mononuclear leukocytes) in vitro (5) and possibly in vivo $(11,12)$. Therefore, we considered the possibility that the presence of macrophages might be a necessary prerequisite for influenza virus infection, viral protein synthesis, and surface antigen expression by autologous lymphocytes. In subsequent experiments, unseparated mononuclear leukocytes were exposed to influenza virus, washed and reincubated (unseparated) for $4 \mathrm{~h}$, and then purified lymphocytes $(>99.5 \%$ ) were obtained by elutriation. After separation, the purified lymphocytes were pulse-labeled $(2 \mathrm{~h})$ with $\left[{ }^{35}\right.$ S]methionine, and cell lysates analyzed as before by SDS PAGE and autoradiography. Results from these experiments demonstrated synthesis of influenza virus proteins by lymphocytes, exposed in the presence of macrophages to infectious virus,

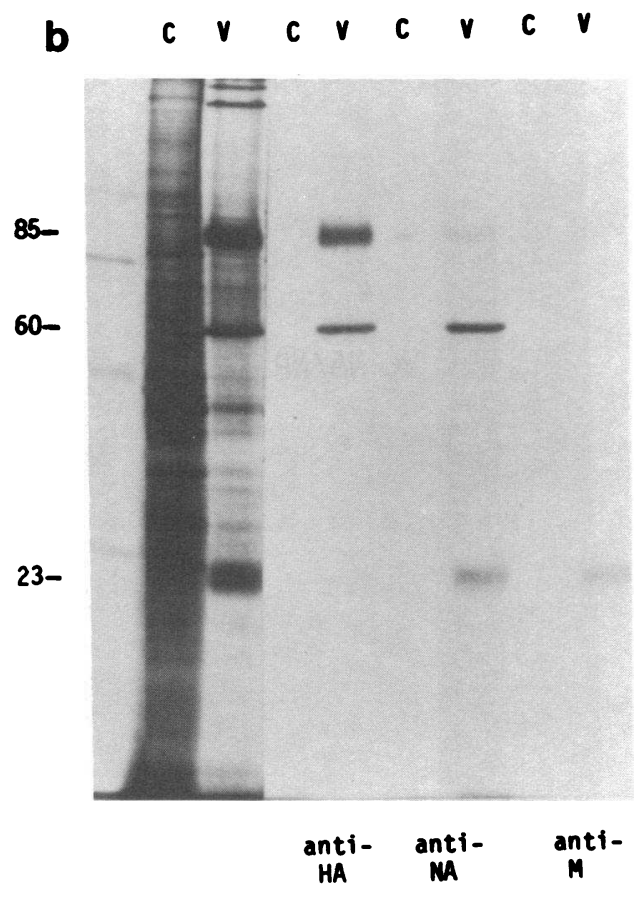

exposed (control, $C$ lanes) or influenza virus-exposed ( $V$ lanes) monocytes-macrophages, using goat polyclonal antisera directed against subtype-specific HA (H1), NA (N1), and M. Lysates were derived from cells pulsed 4-6 $\mathrm{h}$ after sham-exposure or exposure to the H1N1 influenza virus strain. Some cross-reactivity between antisera for the different influenza proteins was noted. 


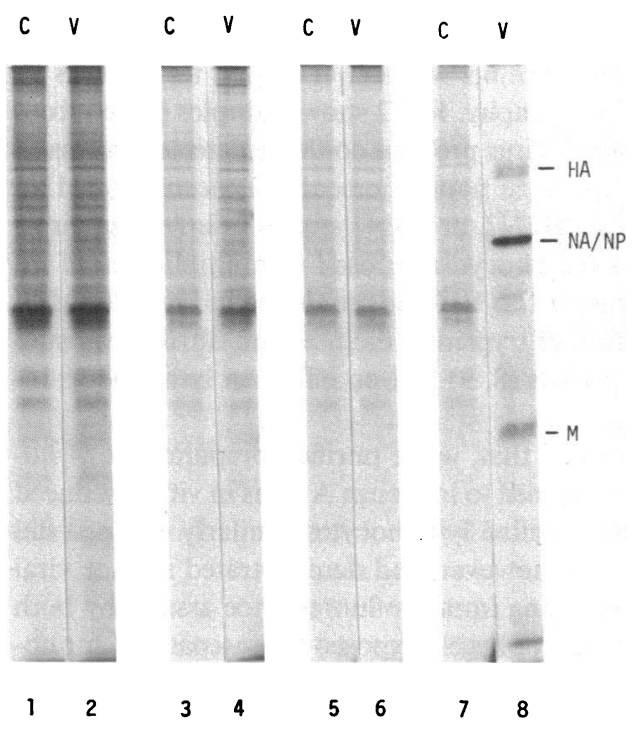

Figure 2. Autoradiograms showing representative results from control ( $C$ lanes) sham-exposed and influenza virus-exposed ( $V$ lanes) lymphocytes obtained by three different techniques, as described in the text. Lanes 1 and 2, lysates from lymphocytes obtained by sequential depletion of adherent cells by passage and culture in plastic dishes; lanes 3 and 4 , lymphocytes obtained by adherence followed by passage of nonadherent cells through nylon wool; lanes 5 and 6 , lymphocytes obtained by countercurrent centrifugal elutriation; and lanes 7 and 8 , lysates from sham-infected (control, $C$ lanes) and influenza virus-infected ( $V$ lanes) macrophages for comparison. and subsequently separated by countercurrent centrifugal elu triation (Fig. $3 a$ ).

A variety of soluble factors produced by macrophages ir response to viral infection (e.g., interferon- $\alpha$ and IL-1) are knowr to be induced by influenza virus $(3,4)$; such factors can havi profound effects on lymphocyte growth and differentiation. $W_{1}$ sought to determine whether soluble factors produced by un infected or influenza virus-infected macrophages would be suf ficient to permit infection of, and subsequent virus antigen syn thesis by, macrophage-depleted lymphocytes. Cell-free super natant fluids from control or influenza virus-infected purifier macrophages were collected $6 \mathrm{~h}$ after infection, the interval afte infection at which maximal influenza protein synthesis had beer noted in both macrophages and lymphocytes. In addition, su pernatant fluids from influenza virus-exposed purified macro phages were collected from 0 to $2 \mathrm{~h}$, from 2 to $4 \mathrm{~h}$ (fresh mediun substituted after $2 \mathrm{~h}$ ), and from 0 to $4 \mathrm{~h}$ after infection. Th fluids from the virus-infected macrophages contained botl IL-1 and interferon activities, measured using standard tech niques $(3,4)$ (data not shown). Purified normal autologous lym phocytes, collected by elutriation, were suspended in the mac rophage-derived supernatant fluids, immediately exposed to in fluenza virus, incubated for $4 \mathrm{~h}$, and pulse-labeled. Cell synthesi was analyzed as before using SDS PAGE and autoradiography No new influenza virus proteins were detected in lymphocyte incubated with any of the supernatant fluids derived from eithe influenza virus-exposed (Fig. $3 b$ shows results using fluid col lected at $6 \mathrm{~h}$ ) or sham-exposed macrophages (data not shown)
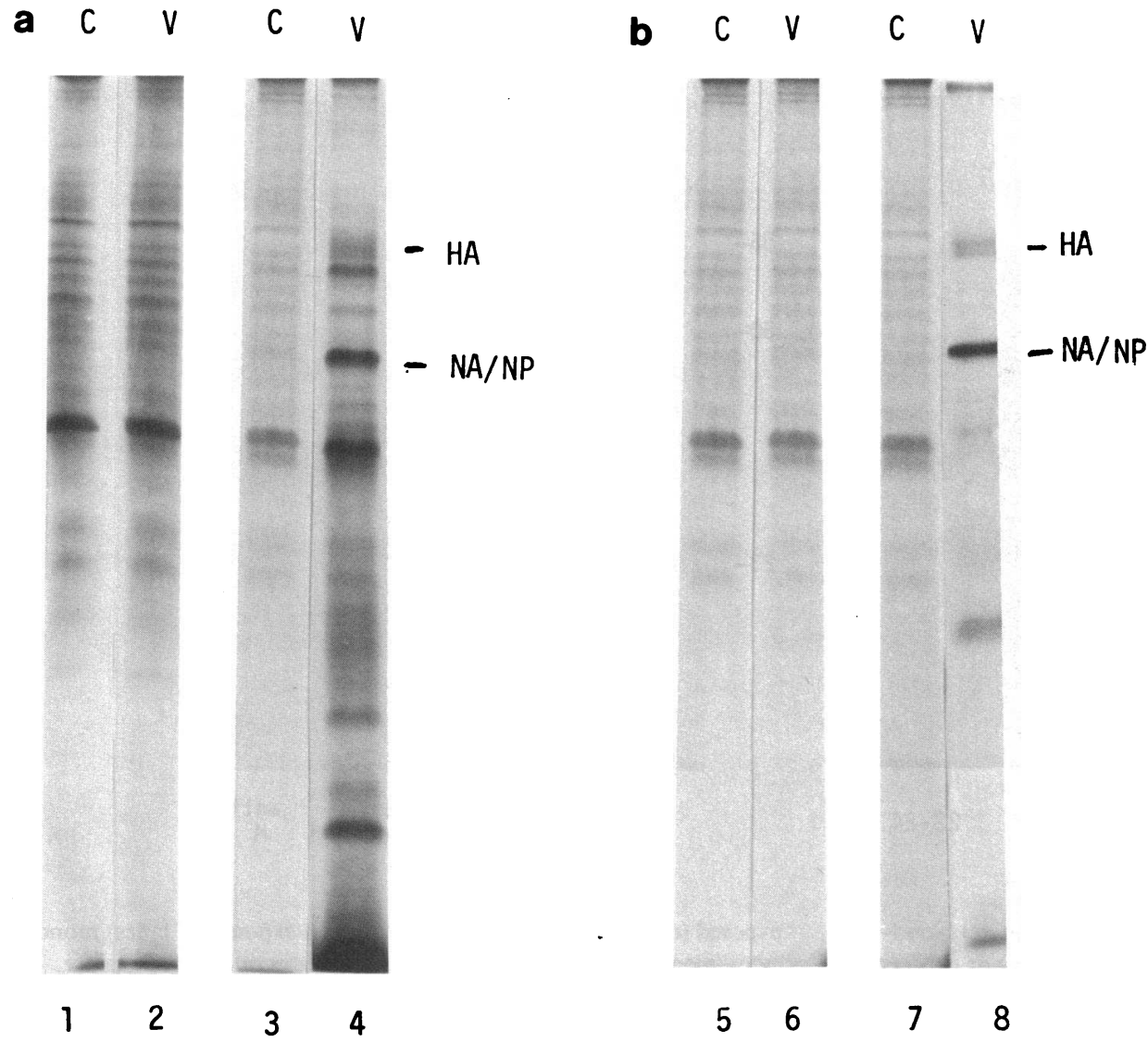

Figure 3. (a, lanes 1-4) Autoradiograms of purified human lymphocyte sham-exposed (control, $C$ lanes) or e: posed ( $V$ lanes) to influenza virus. In fluenza viral HA and NA/NP synthe sis by lymphocytes, 4-6 $\mathrm{h}$ after exposure to influenza A/AA/Marton/43 $\mathrm{H} 1 \mathrm{~N} 1$ in the absence (lanes 1 and 2 ) or presence (lanes 3 and 4 ) of monocytes. The purified lymphocytes (obtained by elutriation) and unseparate mononuclear leukocytes (lymphocytı plus monocytes) were sham-exposed or exposed to virus for $1 \mathrm{~h}$, washed, and reincubated for $4 \mathrm{~h}$. Cells were rapidly chilled to $4^{\circ} \mathrm{C}$, and purified (>99.5\%) lymphocytes were obtaine by elutriation. Purified lymphocytes were then pulse-labeled for $2 \mathrm{~h}$ with $\left[{ }^{35}\right.$ S]methionine, lysed with detergen 1 buffers, and analyzed by SDS PAGE (b, lanes 5-8) Absence of influenza $v$ rus protein synthesis in elutriation-pı rified lymphocytes sham-exposed $(C$. or exposed $(V)$ to influenza virus. Lymphocytes were incubated with cell-free supernatant fluids derived from influenza virus-infected macro phages, immediately exposed to viru and assayed for protein synthesis (lanes 5 and 6). Data are shown for lymphocytes pulsed from 4-6 $\mathrm{h}$ after sham-exposure ( $C$ lanes) or exposure

( $V$ lanes) to influenza virus in the presence of the macrophage-derived supernatant fluid. Lanes 7 and 8 , protein synthesis by control ( $C$ lanes) anı infected ( $V$ lanes) macrophages for comparison. 


\section{Discussion}

The facilitation of influenza virus infection of human lymphocytes by autologous macrophages represents a novel accessory cell function for monocytes-macrophages. Our findings may be analogous to previous reports detailing a requirement for accessory cells, bearing class II histocompatibility (MHC) antigens, for T-lymphocyte proliferative responses to both soluble antigens $(13,14)$, and mitogens (15-18). In a recent study (19), a complete lymphocyte proliferative response to the mitogen phytohemagglutinin (PHA) could be achieved only in the presence of macrophages; addition of macrophage-conditioned supernatant fluids with lymphocyte-activating factor (IL-1) activity, or purified IL1 , led only to partial reconstitution of lymphocyte proliferative responses. The current data suggest that cell-free supernatant fluids from influenza virus-infected macrophages can not per se promote viral protein synthesis by autologous virus-exposed lymphocytes; the data suggest that another, currently undefined, interaction between macrophages and lymphocytes is necessary. The current studies, using autologous leukocytes, do not indicate whether identity of class II MHC antigens is required. In addition, these results do not exclude the possibility that labile factors, elaborated by the macrophages and effective only at very short range, play a role in influenza virus infection of lymphocytes within the microenvironment.

Several studies (20-22), using both light and scanning electron microscopic techniques, have observed that direct physical interaction between antigen-pulsed macrophages and antigenspecific $\mathrm{T}$ lymphocytes occurs in culture within $4 \mathrm{~h}$ and is critical for the subsequent activation of the lymphocytes. We suspect that similar cell-cell interaction may permit transfer of influenza virus RNA from macrophage to lymphocyte, either directly via the broad base observed in macrophage-lymphocyte clusters (20), or indirectly via macrophage endocytosis of virus followed by as yet undefined internal handling events (23), recycling of part or all of the virus genome to the cell surface, and subsequent transfer to the adjoining lymphocyte, possibly via endocytosis by the lymphocyte of its own histocompatibility class II (24) and virus-occupied influenza virus receptor. Others have shown, by electron microscopic autoradiography, transfer of radiolabeled RNA from normal murine macrophages to autologous lymphocytes, with transfer enhanced by exposure of the lymphocytes to endotoxin-stimulated radiolabeled macrophages (25). The current data do not exclude the possibility that cell-to-cell transfer of influenza virus does not occur, but that virus infection of lymphocytes is the result of direct infection of the lymphocyte, which is facilitated in some other way by the presence of adjacent macrophages. However, the viral proteins described in these studies were not merely input proteins, processed and transferred from macrophages to lymphocytes. After infection in the presence of macrophages, subsequent synthesis of viral proteins occurred in purified lymphocyte preparations from which the macrophages had been removed. Thus, viral RNA can be assumed to have been present in the lymphocytes.

The expression of influenza virus HA, NA, and $M$ on the surface of both unstimulated peripheral blood monocytes and lymphocytes, infected together in culture, was demonstrated previously using polyclonal antisera (5). The use of such immunofluorescent techniques did not exclude the possibility that input virus antigens were merely processed and expressed without new antigen synthesis. The current studies extend those observations and demonstrate that the expressed influenza virus an- tigens do represent products of new synthesis by both monocytes and lymphocytes, and further demonstrate a critical requirement for human monocytes in permitting influenza $A$ virus infection of autologous human lymphocytes.

The surface expression of viral antigens by lymphocytes, perhaps coupled with histocompatibility determinants, might be expected to provide an effective stimulus for the activation of influenza virus-specific effector cells, such as cytotoxic Tlymphocytes. Infection of local and/or circulating immune cells by viruses may stimulate production of interleukins, interferons, and other immunoregulatory mediators that may be important for amplification and regulation of host antiviral defense. Because the normal course of events is recovery from, and immunity to, homotypic influenza virus, without substantial sequelae, it may be reasonable to assume that macrophage facilitation of lymphocyte infection represents a component of the immune defense of the host. This would provide an additional basis for our recognition of the importance of macrophage-lymphocyte clusters in the generation of antigen-specific immune responses (26). It remains to be determined whether macrophage facilitation of virus infection of lymphocytes is a general phenomenon, involving other viruses in addition to influenza.

\section{Acknowledgments}

This work was supported by grants AI-15547 and CA-11198 from the National Institutes of Health. D. J. Mock was supported by a fellowship from the National Cancer Cytology Center. F. Domurat is a Wilmot Cancer Research Fellow. M. Licht was supported by a training grant (HL-07496) from the National Institutes of Health.

\section{References}

1. Roberts, N. J., Jr., and R. T. Steigbigel. 1978. Effect of in vitro virus infection on response of human monocytes and lymphocytes to mitogen stimulation. J. Immunol. 121:1052-1058.

2. Roberts, N. J., Jr. 1982. Different effects of influenza virus, respiratory syncytial virus, and Sendai virus on human lymphocytes and macrophages. Infect. Immun. 35:1142-1146.

3. Roberts, N. J., Jr., R. G. Douglas, Jr., R. M. Simons, and M. E. Diamond. 1979. Virus-induced interferon production by human macrophages. J. Immunol. 123:365-369.

4. Roberts, N. J., Jr., A. H. Prill, and T. N. Mann. 1986. Interleukin 1 and interleukin 1 inhibitor production by human macrophages exposed to influenza virus or respiratory syncytial virus: respiratory syncytial virus is a potent inducer of inhibitor activity. J. Exp. Med. 163:511-519.

5. Roberts, N. J., Jr., and P. K. Horan. 1985. Expression of viral antigens after infection of human lymphocytes, monocytes, and macrophages with influenza virus. J. Infect. Dis. 151:308-313.

6. Wahl, L. M., I. M. Katona, R. L. Wilder, C. C. Winter, B. Haraoui, I. Scher, and S. M. Wahl. 1984. Isolation of human mononuclear cell subsets by counterflow centrifugal elutriation (CCE). I. Characterization of B-lymphocyte-, T-lymphocyte-, and monocyte-enriched fractions by flow cytometric analysis. Cell. Immunol. 85:373-383.

7. Keng, P. C., C. K. N. Li, and K. T. Wheeler. 1981. Characterization of the separation properties of the Beckman elutriator system. Cell. Biophysics. 3:41-56.

8. Lazarowitz, S. G., and P. W. Choppin. 1975. Enhancement of the infectivity of influenza $A$ and $B$ viruses by proteolytic cleavage of the hemagglutinin polypeptide. Virology. 68:440-454.

9. Klenk, H.-D., R. Rott, M. Orlich, and J. Blodorn. 1975. Activation of influenza A viruses by trypsin treatment. Virology. 68:426-439.

10. Laemmli, U. K. 1970. Cleavage of structural proteins during the assembly of the head of bacteriophage T4. Nature (Lond.). 227:680-685.

11. Wilson, A. B., D. N. Planterose, J. Nagington, J. R. Park, R. D. 
Barry, and R. R. A. Coombs. 1976. Influenza A antigens on human lymphocytes in vitro and in vivo. Nature (Lond.) 259:582-584.

12. Rukavishnikova, G. E., and A. K. Alekseeva. 1977. Influenza virus antigens in human leukocytes after oral administration of live tissue culture influenza A monovaccine. Acta Virol. (Prague) (Engl. Ed.). 21: 391-396.

13. Oppenheim, J. J., B. G. Leventhal, and E. M. Hersh. 1968. The transformation of column-purified lymphocytes with nonspecific and specific antigenic stimuli. J. Immunol. 101:262-270.

14. Seeger, R. C., and J. J. Oppenheim. 1970. Synergistic interaction of macrophages and lymphocytes in antigen-induced transformation of lymphocytes. J. Exp. Med. 132:44-65.

15. Rosenstreich, D. L., J. J. Farrar, and S. Dougherty. 1976. Absolute macrophage dependency of $T$ lymphocyte activation by mitogens. $J$. Immunol. 116:131-139.

16. Habu, S., and M. C. Raff. 1977. Accessory cell dependence of lectin-induced proliferation of mouse T lymphocytes. Eur. J. Immunol. 7:451-457.

17. Thiele, D. L., M. Kurosaka, and P. E. Lipsky. 1983. Phenotype of the accessory cell necessary for mitogen-stimulated $T$ and $B$ cell responses in human peripheral blood: delineation by its sensitivity to the lysosomotropic agent, L-leucine methyl ester. J. Immunol. 131:22822290.

18. Hünig, T., M. Loos, and A. Schimpl. 1983. The role of accessory cells in polyclonal $\mathrm{T}$ cell activation. I. Both induction of interleukin 2 production and of interleukin 2 responsiveness by concanavalin $\mathrm{A}$ are accessory cell dependent. Eur. J. Immunol. 13:1-6.
19. Williams, J. M., B. J. Ransil, H. M. Shapiro, and T. B. Strom. 1984. Accessory cell requirement for activation antigen expression and cell cycle progression by human T lymphocytes. J. Immunol. 133:29862995.

20. Werdelin, O., O. Braendstrup, and E. Pedersen. 1974. Macrophage-lymphocyte clusters in the immune response to soluble protein antigen in vitro. I. Roles of lymphocytes and macrophages in cluster formation. J. Exp. Med. 140:1245-1259.

21. Nielsen, M. H., H. Jensen, O. Braendstrup, and O. Werdelin. 1974. Macrophage-lymphocyte clusters in the immune response to soluble protein antigen in vitro. II. Ultrastructure of clusters formed during the early response. J. Exp. Med. 140:1260-1272.

22. Braendstrup, O., O. Werdelin, E. M. Shevach, and A. S. Rosenthal. 1979. Macrophage-lymphocyte clusters in the immune response to soluble protein antigen in vitro. VII. Genetically restricted and nonrestricted physical interactions. J. Immunol. 122:1608-1613.

23. Unanue, E. R., D. I. Beller, C. Y. Lu, and P. M. Allen. 1984. Antigen presentation: comments on its regulation and mechanism. $J$. Immunol. 132:1-5.

24. Pernis, B. 1985. Internalization of lymphocyte membrane components. Immunol. Today. 6:45-49.

25. Jonas, L., P. Dörfling, M. Barten, and D. Tessmann. 1976. Autoradiographische untersuchungen zur frage des transfers markierter RNS von makrophagen auf lymphocyten. Acta Histochem. 56:301-308.

26. Lipsky, P., and A. S. Rosenthal. 1975. Macrophage-lymphocyte interaction. II. Antigen-mediated physical interactions between immune guinea pig lymph node lymphocytes and syngeneic macrophages. J. Exp. Med. 141:138-154. 